\title{
The appropriate use of inotropes in shock
}

Myer H. Rosenthal MD

Shock is best defined as a syndrome of failure of the heart to pump blood into the aorta in sufficient quantity or under sufficient pressure to maintain the pressure-flow relationship for adequate tissue perfusion. The various therapeutic options available are best selected to insure the most efficient correction of identifiable pathophysiology. One such approach utilizes the principles of cardiac function outlined by Ernest Starling at the turn of the century. ${ }^{1}$ Using the concept in Figure 1, the clinician attempts to identify the dynamic state of the myocardium hypothesizing the existence of a hyperdynamic contractile heart as might exist with sepsis or a hypodynamic state as seen in cardiogenic shock. Beyond this must be estimated the preload or end-diastolic volume further influencing ventricular output. Preload serves as an indication of adequacy of intravascular volume, yet varies dependent on the contractile state of the myocardium. Thus, in Figure 1 a number of points is designated on the Starling curves depicting states of altered preload and/or contractility. Once assessment of the patient in shock leads to an approximation of this existing pathophysiology selection of therapy follows. Figure 2 outlines the various therapeutic options available dependent on the identified abnormalities. Rapid assessment of response to the chosen therapy must be made to either insure the correctness of the initial selection or to indicate the need for alternative treatment.

Inotropic therapy to increase myocardial contractility is commonly selected. Many considerations are involved in the timing for inotropic stimulation, the correct selection of agent, the desirability for concurrent pharmacology (i.e., afterload reduction) and the proper limits for increase in cardiac output. The goal of increased ventricular output first requires optimal levels of preload. Inotropic stimulation in the presence of intravascular hypovolaemia often fails to achieve increased stroke volume but rather results in undesirable effects of increased afterload, tachycardia and dysrhythmias. Achieving pulmonary artery wedge pressures in excess of $10-12 \mathrm{mmHg}$ and preferably between 15 and $18 \mathrm{mmHg}$ should precede institution of pharmacological inotropic stimulation. The relationship of afterload as indicated by calculation of vascular resistance should also be understood. Afterload reduction with arterial dilator therapy may be preferable to inotropic drugs when systemic arterial pressure is adequate, as such therapy may reduce myocardial wall tension and myocar-

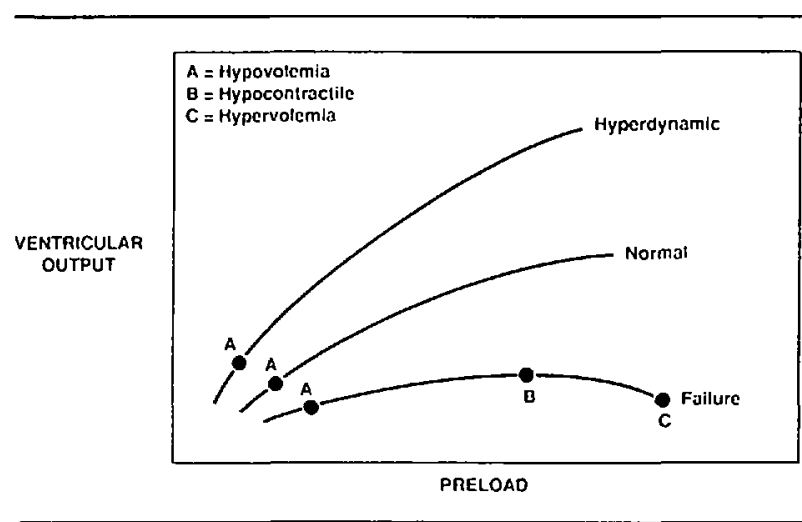

FIGURE I The Starling mechanism used to cstimate the pathophysiology of hacmodynamic insufficiency.

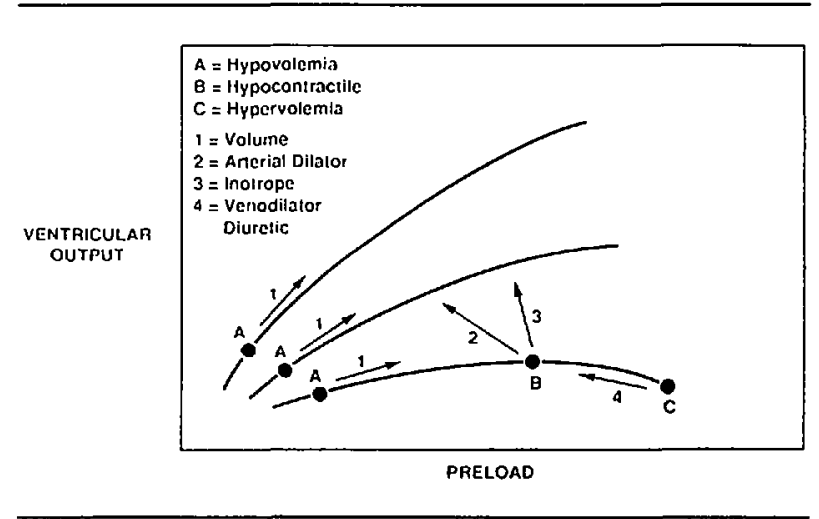

FIGURE 2 Therapy for haemodynamic insufficiency based on estimations of pathophysiologic mechanisms.

dial oxygen demand. Inotropic augmentation in the presence of persistent elevation in afterload may further increase this oxygen demand with resultant ischaemia. In the hypotensive patient with elevated vascular resistance, inotropic therapy may be necessary to provide sufficient blood pressure to allow for institution of vasodilator therapy.

A variety of agents exists having inotropic capability and preference often exists from clinician to clinician and institution to institution. Although one's bias will often

Medical Director of Intensive Care, Stanford University Medical Center, Stanford, California. 
TABLE Inotropic agents

\begin{tabular}{ll}
\hline $\begin{array}{l}\text { Adenyl cyclase } \\
\text { stimulation }\end{array}$ & $\begin{array}{l}\text { Phosphodiesterase } \\
\text { inhibition }\end{array}$ \\
\hline $\begin{array}{l}\text { Dopamine } \\
\text { Epinephrine }\end{array}$ & $\begin{array}{l}\text { Amrinone } \\
\text { Dobutamine }\end{array}$ \\
$\begin{array}{l}\text { Miscellaneous } \\
\text { Isoproterenol }\end{array}$ & - Digitalis \\
\hline
\end{tabular}

determine selection based on beneficial past experience, variations in the pharmacological properties of the different inotropes should be considered. The Table lists the commonly used drugs. Dopamine, epinephrine, dobutamine and isoproterenol stimulate adenyl cyclase to increase production of cyclic-AMP and are thus acknowledged beta-adrenergic stimulators. Amrinone also increases cyclic-AMP, interfering with its metabolism by selectively inhibiting cyclic-AMP phosphodiesterase. The resultant increase in cyclic-AMP provided by these agents produces an increase in intracellular ionized calcium as the pathway for the increase in the force of muscle contraction. Calcium itself as either chloride or gluconate is often administered to increase directly intracellar ionized calcium. The mechanism of action of the digitalis preparations has been debated for many years and now appears to be due to inhibition of $\mathrm{Na}^{+}-\mathrm{K}^{+}$ ATPase with resultant decrease in the $\mathrm{Na}^{+}-\mathrm{K}^{+}$pump and increase in intracellular ionized calcium.

\section{Dopamine}

Dopamine has the capability to stimulate three adrenergic receptors in a dose-related manner. In doses of 2-3 $\mu \mathrm{g} \cdot \mathrm{kg}^{-1} \cdot \mathrm{min}^{-1}$ dopamine has a vasodilating effect on the afferent renal arterioles. ${ }^{2}$ As a result of this dopaminergic stimulation renal blood flow increases with resultant increase in glomerular ultrafiltration. This mechanism has been shown to prevent alpha vasoconstriction mediated increases in renal vascular resistance and decreased renal blood flow observed during infusions of norepinephrine. ${ }^{3}$ Indeed combining dopamine at these doses with vasopressors may increase renal blood flow in shock. Dopamine itself may result in decreased renal blood flow in doses greater than $7 \mu \mathrm{g} \cdot \mathrm{kg}^{-1} \cdot \mathrm{min}^{-1}$ due to its own alpha-1 stimulation. ${ }^{4}$ Dopamine's inotropic effect appears to predominate in doses of $3-10 \mu \mathrm{g} \cdot \mathrm{kg}^{-1} \cdot \mathrm{min}^{-1}$ with alpha effects occurring with doses exceeding $10 \mu \mathrm{g} \cdot \mathrm{kg}^{-1}$. $\mathrm{min}^{-1}$. Although dopamine is often the first drug chosen for inotropic stimulation, it shows poor response in patients with septic shock. Such poor responsiveness to dopamine may be explained by a decrease in the sensitivity of beta-receptors in patients with gram negative septicaemia. ${ }^{5}$ Dopamine beta-hydroxylase activity has also been shown to be decreased in septic shock. ${ }^{6}$ It is responsible for the metabolism of dopamine to the more active compounds norepinephrine and epinephrine, and the diminished activity of this enzyme may play a role in dopamine's poor effect in the presence of sepsis. Adverse effects of dopamine are those related to alpha mediated vasoconstriction with resultant hypoperfusion, organ failure, peripheral ischaemia and acidosis. Excess beta stimulation may also result in tachydysrhythmias and myocardial ischaemia.

\section{Epinephrine}

The effects of epinephrine reflect both beta- and alphaadrenergic stimulation and are dose-related. Long feared because of misuse and the inability to monitor its effects accurately epinephrine is presently preferred by many clinicians for its more potent and predictable inotropic effect. Careful titration with doses between 20 and 100 $\mathrm{ng} \cdot \mathrm{kg}^{-1} \cdot \mathrm{min}^{-1}$ produces a predominant beta inotropic response. Although certainly not free from tachycardic and afterload effects, restriction to the above dose results in far less of these side-effects than expected. Indeed, tiie failure of dopamine to augment cardiac output satisfactorly in septic shock has led many to select epinephrine in preference for this condition. There also may be some rationale, although not yet studied, to use epinephrine with dopamine $\left(2-3 \mu \mathrm{g} \cdot \mathrm{kg}^{-1} \cdot \mathrm{min}^{-1}\right)$ in low cardiac output syndromes in preference to higher doses of dopamine $\left(>7 \mu \mathrm{g} \cdot \mathrm{kg}^{-1} \cdot \mathrm{min}^{-1}\right)$ alone to improve renal perfusion further. ${ }^{7}$

\section{Dobutamine}

Enthusiasm for this agent has been based on its predominant beta effect. ${ }^{8,9}$ Unlike dopamine and epinephrine, increasing doses of dobutamine do not result in alpha adrenergic mediated vasoconstriction. A major effect of dobutamine is a decrease in systemic vascular resistance. Although desirable as a means to increase afterload such an effect may be hazardous in the hypotensive patient where its vascular depressant effect may predominate. The usual dose of dobutamine is $5-20 \mu \mathrm{g} \cdot \mathrm{kg}^{-1} \cdot \mathrm{min}^{-1}$. Experience shows that at higher dosages $\left(>15 \mu \mathrm{g} \cdot \mathrm{kg}^{-1}\right.$. $\mathrm{min}^{-1}$ ) chronotropic and dysrhythmic potential arises. Care must also be exercised in assuring maintenance of preload as with other vasodilator therapy.

\section{Isoproterenol}

Isoproterenol is a direct-acting beta adrenergic agent. It results in increased heart rate, increased cardiac output, and decreased systemic vascular resistance; blood pressure may increase or decrease depending upon baseline haemodynamics. Isoproterenol is generally used for its chronotropic effect in the treatment of hypotension associated with heart block or sinus bradycardia. The 
major adverse effects of isoproterenol are tachydysrhythmias and myocardial ischaemia.

\begin{abstract}
Amrinone
This recently released agent has both positive inotropic and vasodilator properties. ${ }^{10,11}$ The agent is a nonglycoside, non catecholamine whose action is not significantly influenced by beta-blockade. Its usual dosage is as an intravenous bolus of $0.50-0.75 \mathrm{mg} \cdot \mathrm{kg}^{-1}$ followed by an infusion of $5-10 \mu \mathrm{g} \cdot \mathrm{kg}^{-1} \cdot \mathrm{min}^{-1}$. As with dobutamine care must be exercised in hypotensive syndromes lest its vasodilator effect results in further hypotension. Its alternative pathway as a phosphodiesterase inhibitor may be beneficial in synergistically augmenting more standard direct-acting beta-adrenergic agents.
\end{abstract}

\section{Digitalis}

Risks of dysrhythmias due to inadvertent toxicity, potassium disequilibrium and acid-base imbalance has resulted in little enthusiasm for the acute use of digitalis preparations as inotropic therapy in shock. Presently acute digitalization is restricted to the management of supraventricular dysrhythmias.

\section{Calcium}

The positive inotropic effect of calcium administration as calcium chloride or calcium gluconate is related to the increased ionized calcium facilitating more active binding of actin and myosin. This results in an increase in myocardial muscle contractility. However, similar action also exists in the vascular smooth muscle of resistance arterioles. Thus, a rise in blood pressure could be equally a result of vasoconstriction as well as an increase in contractility. Such vasoconstriction and its resultant increase in vascular resistance has the detrimental effect of increased afterload and myocardial oxygen demand. Further concern has recently been raised regarding the role of calcium-induced vasospasm as an aetiology of post-ischaemic organ dysfunction. ${ }^{12}$ Less enthusiasm therefore exists for the previously common practice of bolus administration of calcium to increase blood pressure in hypotensive states and following cardiopulmonary bypass. When used calcium is most often administered as a $100-250 \mathrm{mg}$ bolus of calcium chloride.

Once a decision is made to use inotropic therapy and the appropriate agent selected, the clinician must determine the end-point of this treatment. Commonly, a rise in cardiac output sufficient to maintain the desired mean arterial pressure is selected. In other situations the end-point may be the cardiac output itself, the clinician preselecting a value that is felt to optimize tissue perfusion. Recently, investigators have suggested a more physiologic approach to the selection of an optimal level of cardiac output for a given clinical situation. ${ }^{13}$ This approach is of particular potential benefit in patients with hyperdynamic (septic) shock. It is often in such cases that the clinician is presented with the dilemma of further increase in cardiac output versus vasoconstrictor therapy to provide a minimally acceptable blood pressure. A recommended approach would favour continued inotropic stimulation to increase cardiac output provided total body oxygen consumption $\left(\dot{\mathrm{VO}}_{2}\right)$ continues. Once $\dot{\mathrm{V}}_{2}$ fails to rise with further elevation in cardiac output, such elevation may be unnecessary. The availability of continuous mixed venous oxygen saturation allows for an indirect monitor for determining optimal cardiac output. As $\dot{\mathrm{VO}}_{2}$ rises with increasing output, mixed venous saturation will remain relatively unchanged until $\dot{\mathrm{VO}}_{2}$ fails to show further increase resulting in a rise in mixed venous saturation. Whether one finds it easier to monitor mixed venous saturation or $\dot{\mathrm{VO}}_{2}$, this concept of increasing cardiac output to the limits of increased oxygen utilization provides a physiological end-point for inotropic support in septic shock that may be preferable to other limits often arbitrarily chosen.

\section{References}

1 Starling $E H$. The Linacre Lecture on the Law of the Heart. Longmans, Green and Co., 1918. London: Longmans, Grcen and Co., 1918.

2 Veda S, Yano S, Sakanashi $M$. In vitro evidence for dopaminergic receptors in human renal artery. J Cardiovasc Pharmacol 1982; 4: 76-81.

3 Schaer GL, Fink MP, Parillo JE. Norepinephrine alone versus norepinephrine plus low-dose dopamine: enhanced renal blood flow with combination pressor therapy. Cit Care Med 1965; 13: 492-6.

4 Beregovich J, Blanchi C, Rubler S et al. Dose-related hemodynamic and renal effects of dopamine in congestive heart failure. Am Heart J 1974; 87: 550- 6.

5 Sibley $D R$, Lefkowitz RJ. Molecular mechanisms of receptor desensitization using the beta-adrenergic receptorcoupled adenylate cyclase system as a model. Nature 1985; 317: 1247-9.

6 Harrari A, Martin E, Bouvier AM et al. Decreased dopamine-beta-hydroxylase activity in scptic shock. Anesthesiology 1979; 5: S155.

7 Turnquest PE, Rosenthal $M H$. Acute renal failure in trauma and critically ill patients. Seminars in Anesthesia 1989; 8: 338-46.

8 Stoner JD, Bolen JL, Harrison DC. Comparison of dobutamine and dopamine in treatment of severe heart failure. Br Heart J 1977; 39: 536-9. 
9 Tuttle RR, Mills J. Dobutamine. Circulation Res 1975; 36: 185-95.

10 LeJemtel TH, Keung E. Sonnenblick EH et al. Amrinone: a new non-glycosidic, non-adrenergic cardiotonic agent effective in the treatment of intractable myocardial failure in man. Circulation 1979; 59: 1098-104.

11 Benotti JR, Grossman W, Braunwald E et al. Hemodynamic assessment of amrinone. N Engl Med 1978; 299 : 1373-7.

12 Wooley JL, Barker GR, Jacobsen WK et al. Effect of calcium entry blocker verapamil on renal ischemia. Crit Care Med 1988; 15: 48-51.

13 Astiz ME, Rackow EC, Falk JL et al. Oxygen delivery and consumption with hyperdynamic septic shock. Crit Care Med 1987; 15: 26-8. 


\section{L'utilisation appropriée des inotropes dans le choc}

Le choc se définirait comme la défaillance du cœur à faire circuler du sang dans l'aorte en quantité suffisante ou sous pression suffisante pour maintenir la relation débitpression nécessaire à une perfusion adéquate des tissus. Les nombreuses alternatives thérapeutiques disponibles sont choisies judicieusement pour assurer une correction efficace du mécanisme pathophysiologique sous-jacent. Une des ces approches utilise le principe de la fonction cardiaque telle que décrite par Ernest Starling au début du siècle.' En employant le concept illustré dans la figure 1 (voir page Slxiv), les cliniciens tentent d'identifier l'état dynamique du myocarde en vérifiant l'existence d'un cœur à contractilité hyperdynamique comme c'est possible dans le choc septique ou un état hypodynamique tel que rencontré dans le choc cardiogénique. En plus, il faut évaluer la précharge ou le volume de fin de diastole, qui eux vont influencer le débit ventriculaire. La précharge sert d'indicateur d'un volume intra-vasculaire suffisant mais elle va varier selon l'état de contractilité myocardique. Ainsi, sur la figure 1 , un certain nombre de points sont inscrits sur les courbes de Starling ; ils décrivent des états de précharge et/ou de contractilité modifiés. Dès que l'évaluation du patient en choc nous a conduit à une approximation de la pathophysiologie existante, dès lors une sélection de thérapie s'en suit. La figure 2 (voir page Slxiv), donne un aperçu des nombreuses alternatives thérapeutiques disponibles selon les anomalies identifiées. La réponse au traitement choisi doit être évaluée rapidement soit pour s'assurer que l'on a fait le bon choix ou pour s'orienter vers une thérapie alternative le cas échéant.

La therapie aux inotropes dans le but d'augmenter la contractilité du myocarde est souvent choisie. Plusieurs facteurs sont à considérer dans la chronologie de la stimulation inotropique: le choix du bon agent, la désirabilité d'une thérapie associée (i.e., réduction de post-charge) et les limites acceptables d'augmentation du débit cardiaque. L'atteinte de l'objectif d'un débit ventriculaire augmenté demande d'abord de s'assurer de niveaux optimaux de précharge. La stimulation inotropique en présence d'une hypovolémie intra-vasculaire va souvent faillir à obtenir un volume d'éjection augmenté et produira des effets indésirables du type post-charge augmentee, tachycardie et dysrythmies. Il faut d'abord que la pression capillaire pulmonaire bloquée soit supérieure à $10-12 \mathrm{mmHg}$ et de préférence atteigne 15 à 18 $\mathrm{mmHg}$ avant de commencer toute thérapie aux inotropes. Il faut aussi évaluer l'effet de la post-charge, telle que mesurée par le calcul de la résistance vasculaire. La réduction de la post-charge avec une thérapie aux vasodilatateurs peut parfois être préférable aux inotropes lorsque le pression artérielle systémique est adéquate, puisqu'un tel traitement peut réduire la tension murale du myocarde et sa consommation en oxygène. L'accroissement de l'inotropie en présence d'une surélévation de la post-charge peut produire une augmentation de cette demande en oxygène, avec comme résultat une ischémie. Chez le patient hypotendu avec résistance vasculaire augmentée, la thérapie aux inotropes peut être nécessaire pour amener une pression artérielle suffisante; elle permettra la mise en route d'une thérapie aux vasodilatateurs.

Il y a un bon nombre d'agents inotropes et la préférence varie souvent de clinicien à clinicien et d'institution à institution. Même si des biais personnels vont souvent orienter la sélection surtout sur la foi d'expériences antérieures satisfaisantes, les variations dans les propriétés pharmacologiques des différents inotropes doivent être prises en considération. La tableau énumère les substances utilisées couramment. La dopamine, l'épinéphrine, la dobutamine et l'isoprotérénol stimulent l'adényl cyclase à produire de l'AMP-cyclique et sont donc reconnus comme des stimulants $\beta$-adrénergiques. L'amrinone augmente elle aussi l'AMP-cyclique, en diminuant son métabolisme par l'inhibition sélective de la phosphodiestérase. L'augmentation consécutive en AMP-cyclique amenée par ces agents va produire une augmentation de calcium ionisé intracellulaire ; ceci sera le mécanisme d'accroissement de la force contractile musculaire. Le calcium lui-même soit en chlorure ou en gluconate est souvent administré pour augmenter directement le calcium ionisé intracellulaire. Le mécanisme d'action des préparations digitaliques a fait l'objet de controverse pendant plusieurs années et maintenant semble être du à l'inhibition de la $\mathrm{Na}^{+}-\mathrm{K}^{+}$ATPase avec comme résultante une diminution de l'activité de la pompe $\mathrm{Na}^{+} \cdot \mathrm{K}^{+}$et une augmentation du calcium ionisé intracellulaire. 
TABLEAU Agents inotropes

\begin{tabular}{ll}
\hline $\begin{array}{l}\text { Stimulation de } \\
\text { l'adényl cyclase }\end{array}$ & $\begin{array}{l}\text { Inhibition de la } \\
\text { phosphodiestérase }\end{array}$ \\
\hline Dopamine & Amrinone \\
Épinéphrine & Autres \\
Dobutaminc & - Digitalc \\
lsoprotérénol & - Calcium \\
\hline
\end{tabular}

\section{La dopamine}

La dopamine a la capacité de stimuler trois récepteurs adrénergiques différents selon la dose administrée. En doses de 2 à $3 \mu \mathrm{g} \cdot \mathrm{kg}^{-1} \cdot \mathrm{min}^{-1}$ la dopamine a un effet vasodilatateur sur les artérioles afférentes du rein. ${ }^{2}$ Il résulte de cette stimulation dopaminergique une augmentation du flot sanguin rénal et de l'ultrafiltration glomérulaire. On a montré que ce mécanisme prévient les augmentations de résistance vasculaire rénale amenées par la vasoconstriction alpha et aussi la diminution du flot sanguin rénal observé pendant les infusions de norépinéphrine. ${ }^{3}$ En fait, la combinaison de vasopresseurs à la dopamine administrée à ces doses peut augmenter le flot sanguin rénal dans le choc. La dopamine elle-même, à des doses supérieures à $7 \mu \mathrm{g} \cdot \mathrm{kg}^{-1} \cdot \mathrm{min}^{-1}$, peut amener des diminutions du flot sanguin rénal à cause de sa capacité de stimuler les récepteurs alpha- $1 .{ }^{4}$ L'effet inotrope de la dopamine semble dominer à des doses de 3 à 10 $\mu \mathrm{g} \cdot \mathrm{kg}^{-1} \cdot \mathrm{min}^{-1}$ et ses effets alpha apparaissent à des doses supérieures à $10 \mu \mathrm{g} \cdot \mathrm{kg}^{-1} \cdot \mathrm{min}^{-1}$. Même si la dopamine est souvent l'agent de premier choix pour une stimulation inotropique, elle est peu efficace chez les patients en choc septique. Cette mauvaise réponse à la dopamine peut être expliquée par la diminution de la sensibilité des récepteurs bêta chez les patients qui ont une septicémie à gram négatif. ${ }^{5}$ On a démontré aussi une diminution de l'activité de la dopamine bêta-hydroxylase dans le choc septique. ${ }^{6}$ Cet enzyme est responsable de la biotransformation de la dopamine en norépinéphrine et épinéphrine, et la diminution de son activité peut jouer un rôle dans son manque d'efficacité en présence de choc septique. Les effets indésirables de la dopamine sont reliés à la vasoconstriction consécutive à son effet alpha, ce qui amène de l'hypoperfusion, de la défaillance ischémique, de l'ischémie périphérique et de l'acidose. Une stimulation bêta excessive peut aussi produire des tachydysrythmies et de l'ischémie du myocarde.

\section{Épinéphrine}

Les effets de l'épinéphrine reflètent son activité bêta et alpha-adrénergique et sont proportionnels à la dose. Longtemps crainte à cause d'une mauvaise utilisation et d'une incapacité à évaluer précisément ses effets, l'épinéphrine est actuellement préférée par beaucoup de clini- ciens à cause de son effet inotrope plus puissant et prévisible. Utilisée judicieusement à des doses entre 20 et $100 \mathrm{ng} \cdot \mathrm{kg}^{-1} \cdot \mathrm{min}^{-1}$, elle produit un effet bêta-inotrope prédominant. Même si elle peut produire quand même de la tachycardie et une augmentation de la résistance périphérique, la restriction de son utilisation aux doses ci-haut décrites produira beaucoup moins d'effets secondaires que prévu. En fait le manque d'efficacité de la dopamine à augmenter le débit cardiaque de façon satisfaisante dans le choc septique en a conduit plusieurs à choisir de préférence l'épinéphrine pour cette condition. Une alternative, non suffisamment étudiée, serait d'utiliser l'épinéphrine avec de la dopamine $\left(2-3 \mu \mathrm{g} \cdot \mathrm{kg}^{-1}\right.$ $\min ^{-1}$ ), dans des conditions à bas débit cardiaque, de préférence à des doses plus élevées de dopamine seule $\left(>7 \mu \mathrm{g} \cdot \mathrm{kg}^{-1} \cdot \mathrm{min}^{-1}\right)$ pour améliorer la perfusion rénale. ${ }^{7}$

\section{La dobutamine}

L'enthousiasme pour cette substance a été fondée sur ses effets dominants bêta ${ }^{8,9} \mathrm{~A}$ la différence de la dopamine et de l'épinéphrine, des doses croissantes de dobutamine n'amènent pas de vasoconstriction successive à une stimulation alpha. Un effet important de la dobutamine est sa capacité de diminuer la résistance vasculaire systémique. Même si cet effet est désirable comme moyen de diminuer la post-charge, il peut être ennuyeux chez le patient hypotendu où son effet dépresseur vasculaire peut dominer. La dose habituelle de dopamine est de 5-20 $\mu \mathrm{g} \cdot \mathrm{kg}^{-1} \cdot \mathrm{min}^{-1}$. L'expérience démontre qu'à des doses plus élevées $\left(>15 \mu \mathrm{g} \cdot \mathrm{kg}^{-1} \cdot \mathrm{min}^{-1}\right)$ les effets chronotropes et dysrythmiques augmentent. I faut aussi surveiller attentivement le maintien de la précharge comme avec tout autre vasodilatateur.

\section{Isoprotérénol}

L'isoprotérénol est un agent bêta-adrénergique à action directe. Il produit une augmentation de la fréquence cardiaque, une augmentation du débit cardiaque et une diminution de la résistance vasculaire systémique; la pression artérielle peut augmenter ou diminuer selon les données hémodynamiques de départ. L'isoprotérénol est habituellement utilisé pour ses effets chronotropes dans le traitement de l'hypotension associée au bloc cardiaque ou à la bradycardie sinusale. Ses effets indésirables sont la tachydysrythmie et l'insuffisance du myocarde.

\section{L'amrinone}

Cet agent relativement nouveau est inotrope positif et vasodilatateur. ${ }^{10,11}$ Cet agent n'est pas un glycoside ni une catécholamine, et son action n'est pas influencée de façon significative par un bloc des récepteurs bêta. La dose habituelle sera un bolus intra-veineux de 500 à 700 $\mu \mathrm{g} \cdot \mathrm{kg}^{-1}$ suivi par une infusion de $5-10 \mu \mathrm{g} \cdot \mathrm{kg}^{-1} \cdot \mathrm{min}^{-1}$. 
Comme dans le cas de la dobutamine, il faut être prudent lorsqu'il y a hypotension, sinon ses effets vasodilatateurs vont produire une augmentation de cette hypotension. Son action médiée par l'inhibition de la phosphodiestérase peut être bénéfique en augmentant de façon synergique l'action directe plus courante des agents bêtaadrénergiques.

\section{La digitale}

Il y a peu d'enthousiasme pour l'utilisation en phase aigüe du choc des préparations digitaliques, comme inotropes ; les risques de dysrythmies à la suite de toxicité inattendue, de déséquilibre du métabolisme du potassium et ions $\mathrm{H}^{+}$, sont responsables de cette situation. Actuellement la digitalisation en phase aigüe est réservée au traitement des dysrythmies supraventriculaires.

\section{Le calcium}

L'effet inotrope positif de l'administration de calcium sous forme de chlorure ou de gluconate provient d'une augmentation de calcium ionisé qui facilite une meilleure liaison entre l'actine et la myosine. Ceci amène une augmentation de la contractilité du myocarde. Cependant, une telle activité se produit aussi sur le muscle lisse des artérioles de résistance. Ainsi, une augmentation de pression artérielle peut provenir aussi bien d'une vasoconstriction que d'une augmentation de contractilité. Une telle vasoconstriction et l'augmentation consécutive de la résistance vasculaire a pour effet indésirable d'augmenter la post-charge et la demande du myocarde en oxygène. On pense aussi maintenant que le vasospasme induit par le calcium participerait à l'étiologie de la dysfonction tissulaire post-ischémique. ${ }^{12}$ Dès lors, il y a moins d'enthousiasme pour l'utilisation de calcium en bolus pour augmenter la pression artérielle chez les hypotendus et après la circulation extra-corporelle. Lorsqu'il est utilisé, le calcium est le plus souvent administré sous forme de bolus de $100-250 \mathrm{mg}$ de chlorure de calcium.

Lorsqu'un clinicien a décidé d'utiliser des inotropes et qu'il a choisi l'agent approprié, il doit aussi déterminer l'objectif de ce traitement. De façon courante une augmentation du débit cardiaque suffisante pour maintenir un niveau choisi de pression artérielle moyenne constitue un tel objectif. Dans d'autres situations, le critère choisi sera la valeur du débit cardiaque lui-même ; le clinicien choisira d'avance une valeur telle qu'elle amènera, selon son jugement, une perfusion tissulaire optimale. Récemment, certains investigateurs ont suggéré une approche plus physiologique à la sélection d'un niveau optimal de débit cardiaque pour une situation clinique donnée. ${ }^{13}$ Cette approche est particulièrement intéressante chez les patients en choc hyperdynamique (septique). C'est souvent dans ces situations que le clinicien est pris devant un dilemne : il doit choisir entre une augmentation du débit cardiaque et une thérapie vasoconstrictrice pour obtenir une pression artérielle minimale acceptable. L'approche recommandée suggérerait de continuer la stimulation inotropique pour augmenter le débit cardiaque tant et aussi longtemps que la consommation d'oxygène elle aussi augmente. Lorsque la $\dot{\mathrm{VO}}_{2}$ cesse d'augmenter avec le débit cardiaque, l'élévation de ce dernier devient moins nécessaire. La disponibilité de la mesure du contenu en oxygène du sang veineux mêlé permet de définir indirectement le débit cardiaque optimal. Comme la $\mathrm{VO}_{2}$ augmente avec le débit cardiaque, la saturation du sang veineux mêlé va demeurer relativement stable jusqu'à ce que la $\mathrm{VO}_{2}$ cesse d'augmenter, ce qui amène une augmentation de la saturation veineuse mêlée. Qu'un clinicien trouve plus facile de mesurer la saturation veineuse mêlée ou la consommation d'oxygène, ce concept d'augmentation du débit cardiaque à la limite de l'augmentation de l'utilisation de l'oxygène, constitute un point de référence physiologique pour le support inotropique dans le choc septique qui peut être préférable à d'autres objectifs souvent choisis arbitrairement.

\section{Références}

(Voir page Slxvi) 